CS 2018.01.01.9

Bionatura Conference Series Vol 1. No 1.2018

Publicación del IV CONGRESO INTERNACIONAL DE BIOTECNOLOGÍA Y BIODIVERSIDAD IV CIBB Y XV FORO INTERNACIONAL BANANERO

INVESTIGATION / RESEARCH

Previos / Index / Next

\title{
Metabolitos presentes en Capsicum chinense en dos estados de maduración cultivados en diferentes tipos de suelos de Yucatán, México
}

Metabolites present in Capsicum chinense of two ripening stages cultivated in different soil types in Yucatan, Mexico

Oney-Montalo J. ${ }^{1}$, López-Domínguez C. ${ }^{1}$, Zamacona-Ruiz M. ${ }^{1}$, Gómez-Rincón E.1, Ramíre-Sucrez M. ${ }^{1}$, Rodríguez-Buenfil I. ${ }^{{ }^{*}}$

Disponible en: http://dx.doi.org/10.21931/RB/CS/2018.01.01.9

\section{RESUMEN}

El chile habanero (Capsicum chinense) es la principal especie hortícola explotada comercialmente en la Península de Yucatán, México. Cuenta con denominación de origen desde el 2010, basado en sus características de sabor, aroma, pungencia, color y vida de anaquel que se encuentran fuertemente ligados con las particularidades del suelo en donde es cultivada la planta. La interacción plantasuelo afecta el desarrollo del fruto y por consiguiente el contenido de algunos metabolitos secundarios. En el presente trabajo se evaluó el efecto del estado de madurez de chiles habaneros crecidos en tres diferentes suelos característicos de la Península de Yucatán México, sobre el contenido de los principales metabolitos presentes en éstos como: capsaicina, dihidrocapsaicina, ácido ascórbico, actividad antioxidante y polifenoles totales. Se empleó un diseño factorial 3 x 2 para los factores tierra (roja, café y negra) y grado de madurez: inmaduro (chile de color verde) y maduro (chile de color naranja). El grado de madurez, fue el factor que 
tuvo efecto sobre todos los metabolitos analizados, mientras que, de tipo de suelo, tuvo efecto en todos excepto en la actividad antioxidante. Existió interacción de los dos factores (grado de madurez y tipo de tierra) para el contenido de capsaicina, dihidrocapsaicina y capsaicinoides totales y Vitamina C. El chile maduro (color naranja) cosechado de plantas crecidas en suelo rojo (K'ankab) fue el que tuvo los más altos contenidos de ácido ascórbico $(113.8 \pm 0.10 \mathrm{mg} / 100 \mathrm{~g})$, capsaicinoides totales $(702.6 \pm 0.00 \mathrm{mg} / 100 \mathrm{~g})$, capsaicina $(319.36 \pm 0.00 \mathrm{mg} / 100 \mathrm{~g})$, dihidrocapsaicina $(383.23 \pm 0.00 \mathrm{mg} / 100 \mathrm{~g})$, polifenoles totales $(90.25 \pm 0.06 \mathrm{mg}$ GAE/100 g) y actividad antioxidante $(95.43 \pm 0.10 \%)$

Palabras clave: Chile habanero, Capsicum chinenese, Capsaicinoides, Polifenoles totales, actividad antioxidante, grado de madurez, tipo de suelo.

\section{ABSTRACT}

Habanero pepper (Capsicum chinense) is the main horticultural specie commercially exploited in the Yucatan Peninsula, Mexico. It has denomination of origin since 2010, based on its characteristics of flavor, aroma, pungency, color and shelf life that are strongly related to the particularities of the soil where the plant is grown. Plant-soil interaction affects the development of the fruit and consequently the content of some secondary metabolites. In the present work the effect of the maturation state of habanero pepper grown in three different soils, representative of the Yucatan Peninsula of Mexico, was evaluated on the content of the main metabolites: capsaicin, dihydrocapsaicin, ascorbic acid, antioxidant activity and total polyphenols. A $3 \times 2$ factorial design was used for the soil factors (red, brown and black) and degree of ripening: unripe (green pepper) and ripe (orange pepper). The degree of ripening was the factor that had effect on all the metabolites analyzed, while the type of soil had an effect on all but the antioxidant activity. There was interaction of the two factors (degree of ripening and type of soil) for the content of capsaicin, dihydrocapsaicin and total capsaicinoids. The ripe pepper (orange color) harvested from plants grown in red soil (K'ankab) was the one that had the highest contents of ascorbic acid $(113.8 \pm 0.10 \mathrm{mg} / 100 \mathrm{~g})$, capsaicin (319.36 $\pm 0.00 \mathrm{mg} / 100 \mathrm{~g})$, dihydrocapsaicin $(389 \pm 0.00 \mathrm{mg} / 100 \mathrm{~g})$, total polyphenols $(90.25 \pm 0.06 \mathrm{mg}$ GAE / $100 \mathrm{~g})$ and antioxidant activity $(95.43 \pm$ $0.10 \%)$ 
El chile habanero (Capsicum chinense jacq) producido en la Península de Yucatán en México, es reconocido internacionalmente por tener una calidad superior a los cultivados en otras partes del mundo, esto debido a su mayor vida de anaquel y a su alta pugnencia. ${ }^{1}$ Estas características, lo han posicionado como la principal especie hortícola explotada actualmente en la Península de Yucatán, convirtiéndola en un referente socio-económico de la región. ${ }^{2}$

El alto contenido de capsaicinoides que posee el chile habanero, es uno de los principales motivos de su gran interés comercial, aunque en los últimos años se han identificado que este fruto posee otros metabolitos secundarios de gran interés para la industria alimentaria y farmacéutica, como diferentes tipos de polifenoles, carotenoides, ácido ascórbico (vitamina C), y tocoferoles. ${ }^{3}$ Estos compuestos fitoquímicos, se caracterizan por sus diversas funciones farmacológicas, mostrando principalmente efecto antioxidante, $y$ potencial actividad anticancerígena. ${ }^{4}$

El contenido bioquímico del fruto, se va a ver influenciado por varios factores como son las condiciones climáticas en las que se lleva a cabo el cultivo y el tipo de genotipo de la planta. El grado de madurez, es otro factor importante que influye en la calidad de la composición fitoquímica del fruto, esto debido a que durante el proceso de maduración este sufre varias modificaciones bioquímicas, fisiológicas y estructurales. ${ }^{5}$ Este chile cuenta con la denominación de origen “Chile habanero de la Península de Yucatán” desde el año $2010^{6}$, basado en sus características de sabor, aroma, pungencia, color y vida de anaquel que se encuentran fuertemente ligados con las particularidades del suelo en donde es cultivada la planta. La interacción planta-suelo afecta el desarrollo del fruto y por consiguiente el contenido de algunos metabolitos secundarios.

Los principales suelos en los que se lleva a cabo el cultivo son el K'ankab (Suelo rojo), el Boxlu'um (suelo negro) y Ch'ich lu'um (suelo café rojizo), dicha clasificación proviene de la lengua maya, y es ampliamente utilizada en la región para referirse a los diferentes tipos de suelos que están presentes en la Península. El K'ankab se caracteriza por ser un suelo rocoso con contenido de materia orgánica menor en comparación con otros suelos de la zona, y con la capacidad de retener menor humedad que el Boxlu'um, el cual se caracteriza por encontrarse en las partes altas del micro relieve, lo cual hace que esta clase de tierra presente una mayor retención de humedad, además de altos contenidos de materia orgánica y fosforo asimilable. El Ch'ich 'lu'um se caracteriza por ser un suelo con grava, 
puede ser de varios colores, café rojizo a negro, además de poseer la capacidad de retener mayor cantidad de humedad. ${ }^{7}$

Debido a lo mencionado anteriormente, el objetivo del presente trabajo fue evaluar el efecto del estado de madurez de chiles crecidos en tres diferentes suelos sobre el contenido de metabolitos como capsaicinoides totales (suma de capsaicina y dihidrocapsaicina), ácido ascórbico, actividad antioxidante y polifenoles totales, a fin de determinar cuál es el suelo más adecuado para el cultivo de esta planta y el estado de madurez del fruto recomendado en dependencia de la concentración de los metabolitos analizados.

\section{MATERIALES Y MÉTODOS}

\section{Materia prima}

Se emplearon chiles habaneros (Capsicum Chinense jacq) maduros e inmaduros (naranjas y verdes) cosechados al mismo tiempo a los 132 días posteriores al trasplante (DPT) de plántulas (45 días de crecidas con $15 \mathrm{~cm}$ de alto y cuatro hojas verdaderas) sembradas en tres tipos de suelo, el K'ankab (suelo rojo), el Boxlu'um (suelo negro) y Ch'ich lu'um (suelo café rojizo) en invernadero con condiciones controladas de riego y fertilización. La temperatura ambiente estuvo en un rango de 24 a $47{ }^{\circ} \mathrm{C}$ con una humedad relativa del $91 \%$.

\section{Secado de chile habanero}

Una vez realizada la cosecha, se llevó a cabo el proceso de secado por la técnica de liofilización. Esta se realizó a una temperatura de $-50{ }^{\circ} \mathrm{C}$ y una presión de 0.200 mBar por $72 \mathrm{~h}$ Posterior al secado de los chiles, se realizó la molienda de ellos para obtener los polvos de cada tipo de muestra, los cuales fueron almacenados en bolsas a temperatura ambiente.

\section{Extracción de polifenoles}

Se pesaron $0.5 \mathrm{~g}$ de cada muestra de chile y se colocaron en tubos cónicos de 15 $\mathrm{mL}$, posteriormente se agregaron $2.5 \mathrm{~mL}$ de una mezcla metanol: agua (80:20) y se sonicaron a $42 \mathrm{KHz}$ durante 30 minutos, después el extracto fue centrifugado a $4700 \mathrm{rpm}$ y $4{ }^{\circ} \mathrm{C}$ durante 30 minutos, para finalmente filtrar el sobrenadante con 
un filtro de membrana de nylon con un tamaño de poro de $0.45 \mu \mathrm{m}$. Estos extractos fueron empleados tanto para la determinación de polifenoles totales como para la determinación de la actividad antioxidante.

\section{Extracción de capsaicinoides y ácido ascórbico (Vitamina C)}

Se pesaron $50 \mathrm{mg}$ de cada muestra de chile y se colocaron en tubos cónicos de 15 $\mathrm{mL}$, posteriormente se agregaron $4 \mathrm{~mL}$ de una mezcla Agua:Acetonitrilo (80:20) y se agitó con ayuda de un vortex, para su homogenización. Se sonicaron los tubos por 20 minutos a $42 \mathrm{KHz}$, para finalmente filtrar la muestra con un filtro de membrana de nylon con un tamaño de poro de $0.45 \mu \mathrm{m}$. Estos extractos fueron empleados tanto para la determinación de capsaicinoides como para la determinación de la vitamina $\mathrm{C}$ como ácido ascórbico.

\section{Determinación de actividad antioxidante}

El método que se usó para calcular el porcentaje de actividad antioxidante es el de inhibición del radical de DPPH (Brand et al. 1995). ${ }^{8}$

\section{Determinación de polifenoles totales}

El método que se usó para cuantificar los polifenoles totales fue el de Folin Ciocalteu. $^{9}$

Las cuantificaciones de algunos de los metabolitos se realizaron por cromatografía de ultrapresion (UPLC) usando estándares de capsaicina, dihidrocapsaicina y ácido ascórbico mientras que para la actividad antioxidante y polifenoles totales fue por espectrofotometría.

\section{Determinación de metabolitos por UPLC}

Se utilizó un equipo UPLC Acquity H Class (Waters, USA) con un detector de arreglo de diodos. Y una columna Acquity UPLC HSS C18 (100 А, $1.8 \mu \mathrm{m}, 2.1 \mathrm{x}$ $50 \mathrm{~mm}$ ) (Waters, USA). 
Las condiciones cromatográficas para el análisis de la Capsaicina e Dihidrocapsaicina, consistieron en una fase móvil isocrática conformada por Acetonitrilo (fase móvil A) y Agua con ácido fórmico al $0.1 \%$ (fase móvil B) en una proporción de 60:40, la velocidad de flujo fue de $0.2 \mathrm{~mL} / \mathrm{min}$, la temperatura de la columna de $27^{\circ} \mathrm{C}$, el volumen de inyección $2 \mu \mathrm{L}$ y la longitud de onda fue de $280 \mathrm{~nm}$. Los capsaicinoides totales fueron reportados como la suma de la capsaicina y la dihidrocapsaicina.

\section{Ácido ascórbico.}

Las condiciones cromatográficas para el análisis consistieron en una fase móvil isocrática conformada por agua con ácido fórmico al $0.1 \%$, con una velocidad de flujo de $0.25 \mathrm{~mL} / \mathrm{min}$, la temperatura de la columna fue de $27^{\circ} \mathrm{C}$, el volumen de inyección $2 \mu \mathrm{L}$ y la longitud de onda fue de $244 \mathrm{~nm}$.

\section{Diseño factorial $3 \times 2$.}

Se realizó un diseño factorial 3 × 2 con la finalidad de evaluar el efecto de los factores sobre la concentración de metabolitos como capsaicinoides totales, vitamina $\mathrm{C}$, polifenoles totales y actividad antioxidante, siendo el factor A el tipo de suelo y el factor B el grado de madurez, los niveles de A eran: rojo (-1), café $(0)$ y negro $(+1)$, mientras que los niveles del factor B eran: Verde $(-1)$ y Naranja $(+1)$.

\section{Análisis de datos}

El análisis de datos se realizó con el paquete estadístico Statgrafics Centurion XVII.II-X64.

\section{RESULTADOS Y DISCUSIONES}

En la Tabla 1 se presentan los resultados obtenidos correspondientes a los principales metabolitos presentes en las muestras de chile habanero, así como el porcentaje de actividad antioxidante. En esta, podemos observar que los chiles maduros (naranjas) fueron los que presentaron en promedio mayor concentración de capsaicinoides, vitamina $\mathrm{C}$, polifenoles y mayor actividad antioxidante en comparación de los chiles inmaduros (verdes). Estos resultados indican que a 
mayor concentración de polifenoles y Vitamina $\mathrm{C}$, se tiene una mayor actividad antioxidante, lo que coincide con lo reportado por Alvarez et al ${ }^{5} .2011$.

\begin{tabular}{|c|c|c|c|c|c|}
\hline $\begin{array}{c}\text { Tipo de } \\
\text { suelo }\end{array}$ & Madurez & $\begin{array}{c}\text { Actividad } \\
\text { antioxidante } \\
(\%)\end{array}$ & $\begin{array}{c}\text { Vitamina C } \\
(\mathbf{m g} / \mathbf{1 0 0 g})\end{array}$ & $\begin{array}{c}\text { Capsaicinoides } \\
\text { totales } \\
(\mathbf{m g} / \mathbf{1 0 0 g})\end{array}$ & $\begin{array}{c}\text { Polifenoles totales } \\
\text { (mg/100g) }\end{array}$ \\
\hline Rojo & Verde & $91.2 \pm 0.3$ & $81.7 \pm 2.2$ & $537.6 \pm 223.6$ & $64.9 \pm 0.7$ \\
\hline Café & Verde & $88.8 \pm 3.2$ & $71.0 \pm 3.0$ & $287.4 \pm 0.0$ & $49.8 \pm 1.3$ \\
\hline Negro & Verde & $90.2 \pm 2.9$ & $87.9 \pm 0.9$ & $247.5 \pm 0.0$ & $53.3 \pm 1.7$ \\
\hline Rojo & Naranja & $95.4 \pm 0.1$ & $113.8 \pm 0.1$ & $702.6 \pm 0.0$ & $90.3 \pm 0.1$ \\
\hline Café & Naranja & $93.3 \pm 2.2$ & $107.0 \pm 1.4$ & $494.0 \pm 0.0$ & $69.2 \pm 3.6$ \\
\hline Negro & Naranja & $91.1 \pm 0.1$ & $84.2 \pm 3.6$ & $444.0 \pm 5.7$ & $73.2 \pm 2.2$ \\
\hline
\end{tabular}

Tabla 1. Resultados obtenidos de la cuantificación de diferentes metabolitos y de la evaluación de la actividad antioxidante en el chile habanero cultivado en diferentes tipos de suelo con diferente grado de madurez.

El ANOVA multifactorial indicó con un $95 \%$ confiabilidad, que, de los 2 factores evaluados, solamente el grado de madurez mostró efecto significativo sobre el porcentaje de actividad antioxidante. En la gráfica de medias correspondiente (Figura 1), se muestra que el máximo valor $(95.43 \pm 0.10 \%)$ se obtuvo en el chile maduro (naranja).

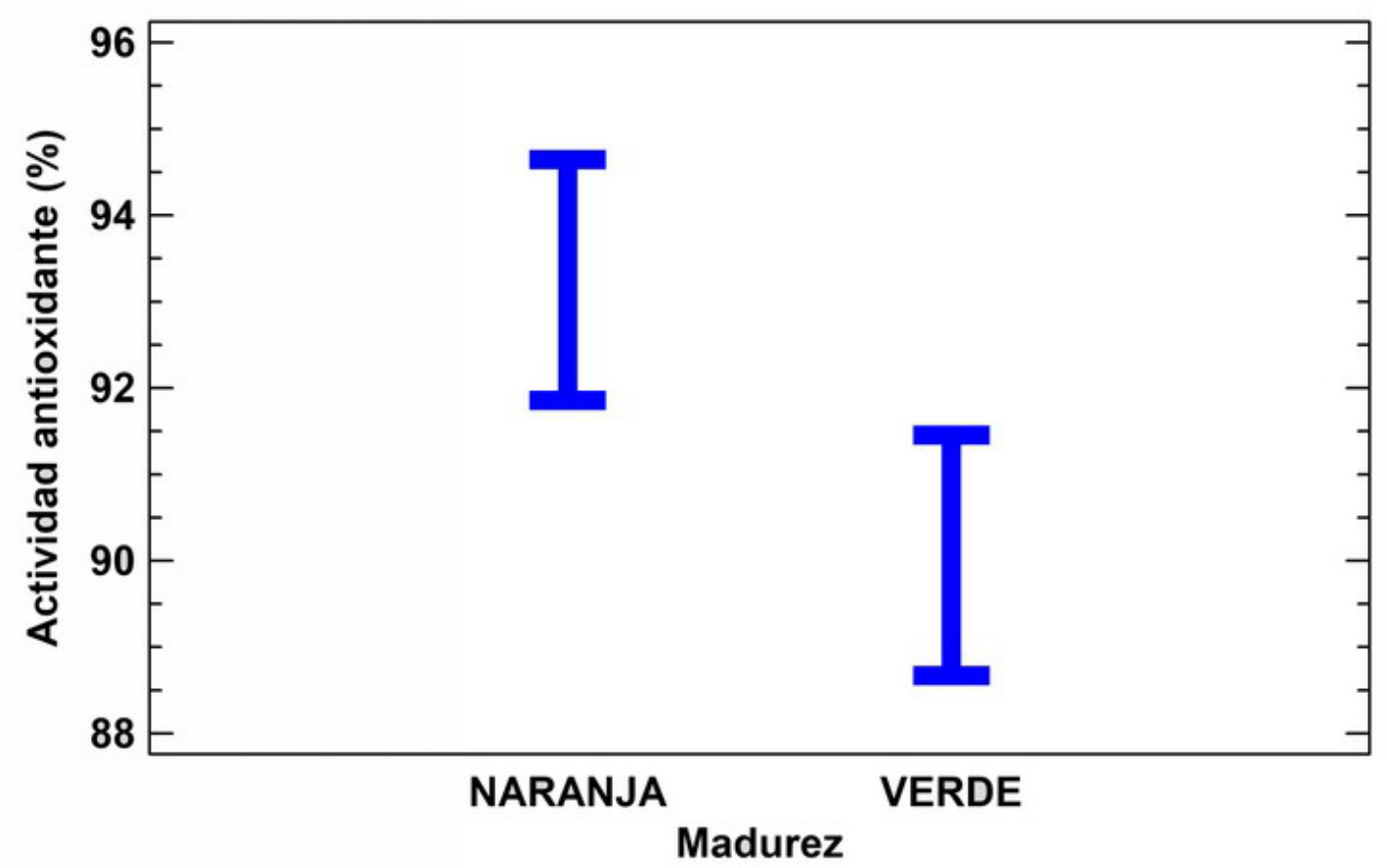


Figura 1. Gráfica de medias para la actividad antioxidante en relación al grado de madurez

En la Figura 2 se representa el diagrama de Pareto obtenido del análisis del diseño experimental, en el que se observa que ambos factores (tipo de suelo y el grado de madurez) tienen efecto significativo sobre la concentración de capsaicinoides totales (suma de capsaicina e dihidrocapsaicina), siendo el tipo de suelo el que presenta mayor efecto sobre la respuesta. El valor más alto tanto de capsaicina $(319.36 \pm 0.00 \mathrm{mg} / 100 \mathrm{~g})$, como de dihidrocapsaicina $389 \pm 0.00 \mathrm{mg} / 100 \mathrm{~g}$, se obtuvo con el chile maduro (naranja) cosechado de plantas crecidas en el suelo rojo. Estos resultados coinciden con el trabajo realizado por Menichini et al. ${ }^{10}$, en el que se reportan concentraciones mayores de capsaicina $(436.3 \mathrm{mg} / 100 \mathrm{~g}$ de chile seco) y de dihidrocapsaicina $(249.8 \mathrm{mg} / 100 \mathrm{~g})$ en el chile habanero maduro en comparación con el inmaduro (107.1 mg/100g de capsaicina y $35.7 \mathrm{mg} / 100 \mathrm{~g}$ de dihidrocapsaicina). Asimismo, ellos obtuvieron una mayor actividad antioxidante en el chile maduro (naranja).

El aumento en la concentración de los capsaicinoides en el fruto maduro (naranja) se puede deber al aumento de la concentración de los ácidos fenólicos durante el proceso de maduración de acuerdo con Howard et al ${ }^{11}$, siendo éstos precursores de la vanillilamina, la cual al unirse al ácido 8-metil-6- nonenoico, forman la capsaicina siendo éste el capsaicinoide mayoritario en Capsicum chinense.

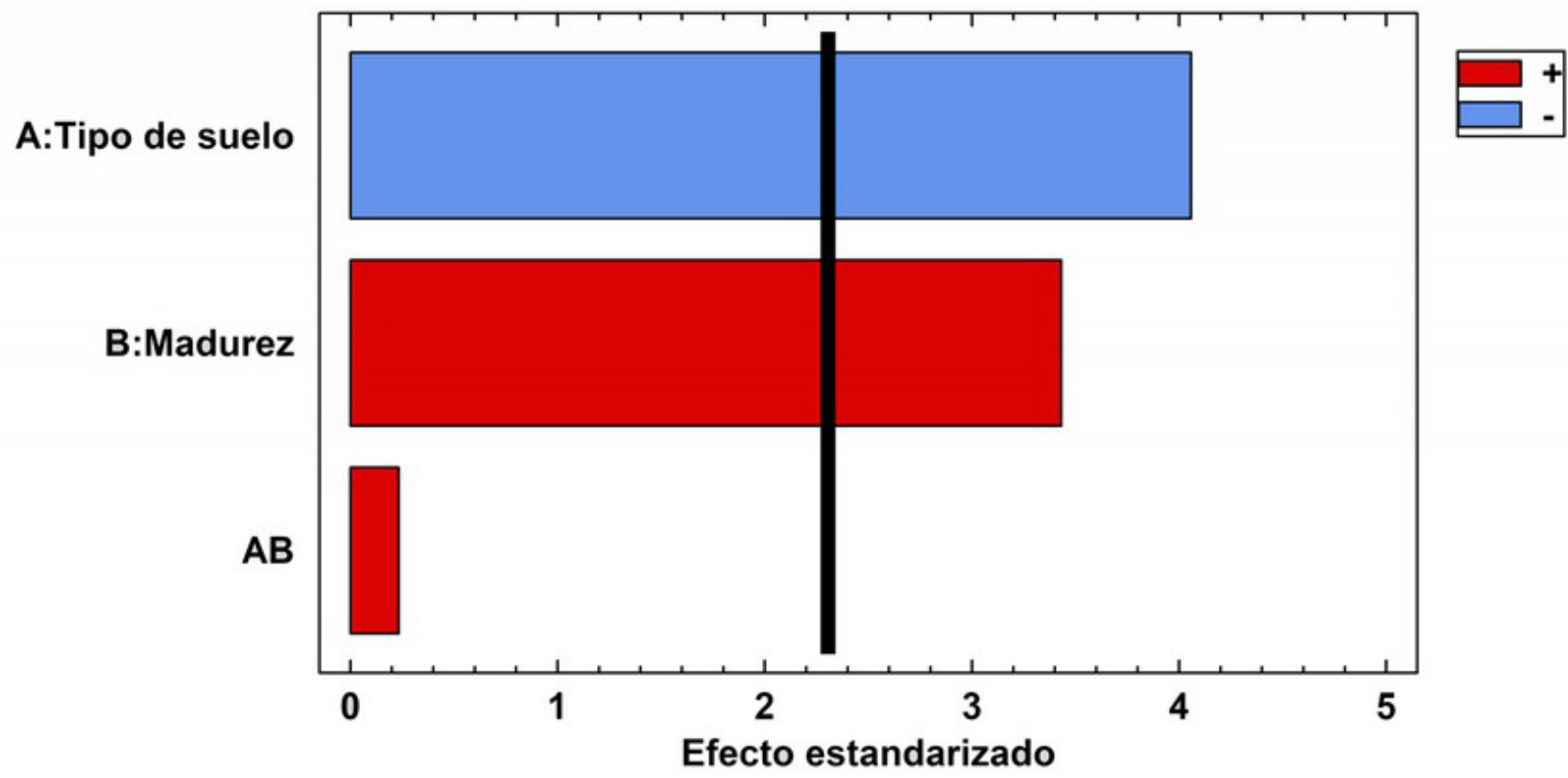

Figura 2. Diagrama de Pareto obtenido del diseño 3 x 2 para evaluar el efecto de los factores sobre la concentración de capsaicinoides totales. 
Mediante un ANOVA multifactorial se evaluó el efecto de los factores (tipo de suelo y el grado de madurez), sobre la concentración de Vitamina C, este nos indicó con un $95 \%$ de confiabilidad que los dos factores evaluados y su interacción tuvieron efecto significativo sobre el contenido de Vitamina C. En la Figura 3 podemos observar el grafico de interacciones obtenido, en el que se puede corroborar que el chile maduro (naranja) cultivado en suelo rojo presentó el valor más alto de Vitamina C (113.8 $\pm 0.10 \mathrm{mg} / 100 \mathrm{~g})$, mostrando diferencias significativas en comparación con las otras combinaciones de los factores. En la literatura se encuentra reportado que conforme incrementa la madurez del chile habanero, se da un aumento en la concentración de Vitamina $\mathrm{C}$ presente en este, tal como lo indica Siddiqui et al. ${ }^{12}$, el cual cuantifico una concentración de 151.0 $\mathrm{mg} / 100 \mathrm{~g}$ de este metabolito en chile maduro (naranja). Mendoza et al. ${ }^{13}$, menciona que la mayor concentración de Vitamina $\mathrm{C}$ en el chile maduro (naranja) está relacionado con el aumento de azucares presentes en el fruto cuando este alcanza su estado maduro (naranja), ya que a partir de estos azucares se lleva a cabo la biosíntesis de vitamina.
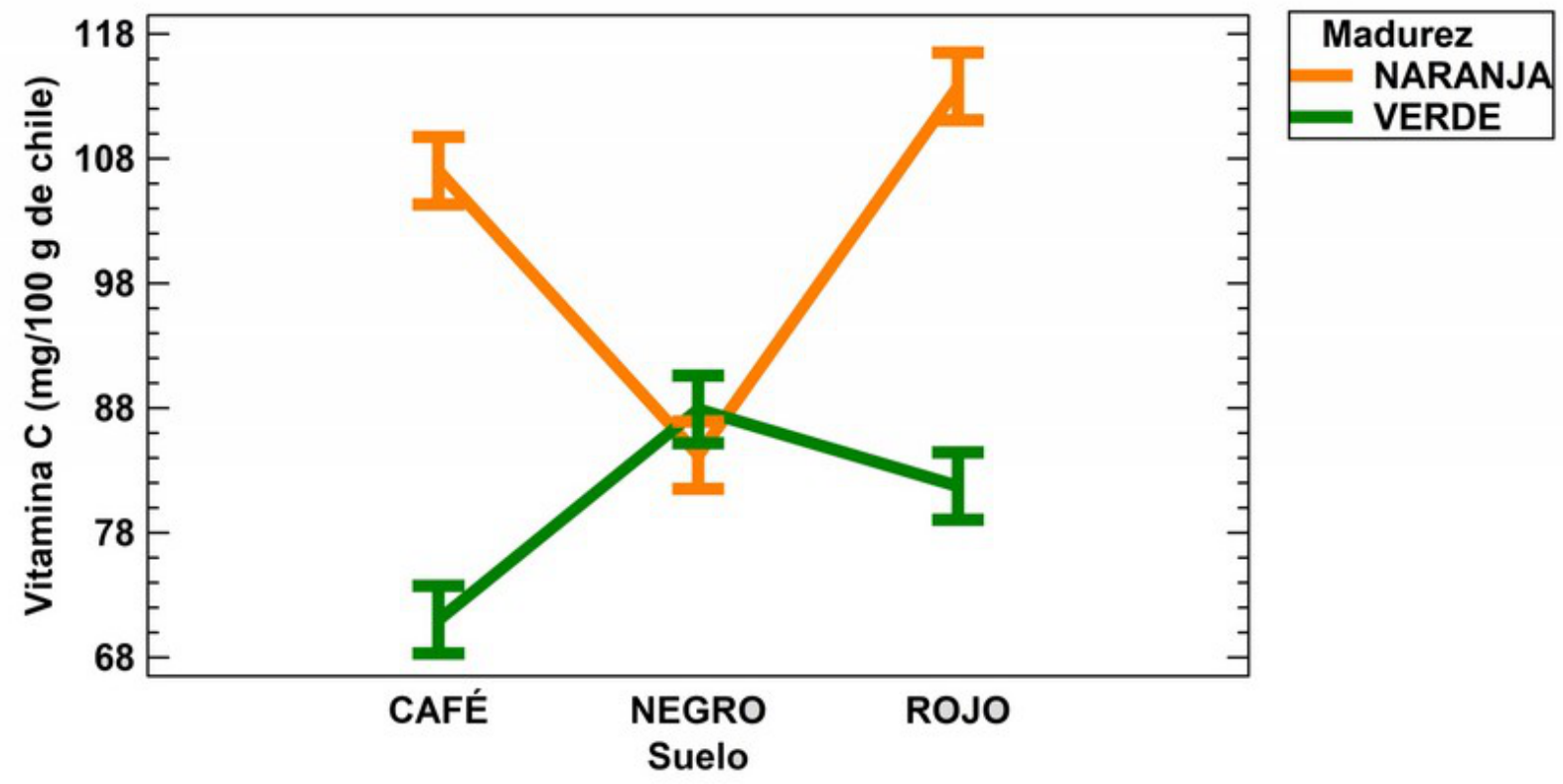

Figura 3. Gráfico de interacciones entre los factores tipo de suelo y grado de madurez con respecto a la concentración de Vitamina $C(\mathrm{mg} / 100 \mathrm{~g}$ de chile seco).

En relación al contenido de polifenoles totales, en la figura 4 se representa el diagrama de Pareto obtenido del análisis del diseño factorial, en el que se observa 
que el grado de madurez es el factor que tiene mayor efecto sobre la concentración de polifenoles totales, seguido del tipo suelo en el que la planta es crecida. La máxima cantidad de polifenoles totales fue de $90.25 \pm 0.06 \mathrm{mg} \mathrm{GAE} / 100 \mathrm{~g}$ en el chile maduro (naranja) cosechado de plantas crecidas en el suelo rojo. Siddiqui et al., ${ }^{12}$ reporta que la concentración de polifenoles totales en el chile habanero incrementa conforme aumenta la madurez de este, registrando para los chiles maduros concentraciones de polifenoles totales de $330.79 \mathrm{mg} \mathrm{GAE} / 100 \mathrm{~g}$ de chile seco. Según lo reportado por Howard et $\mathrm{al}^{11}$, conforme se desarrolla el proceso de maduración, los flavonoides presentes en el chile se convierten en diferentes tipos de ácidos fenólicos, incrementando la concentración de estos, y contribuyendo a un aumento en la concentración de polifenoles totales.

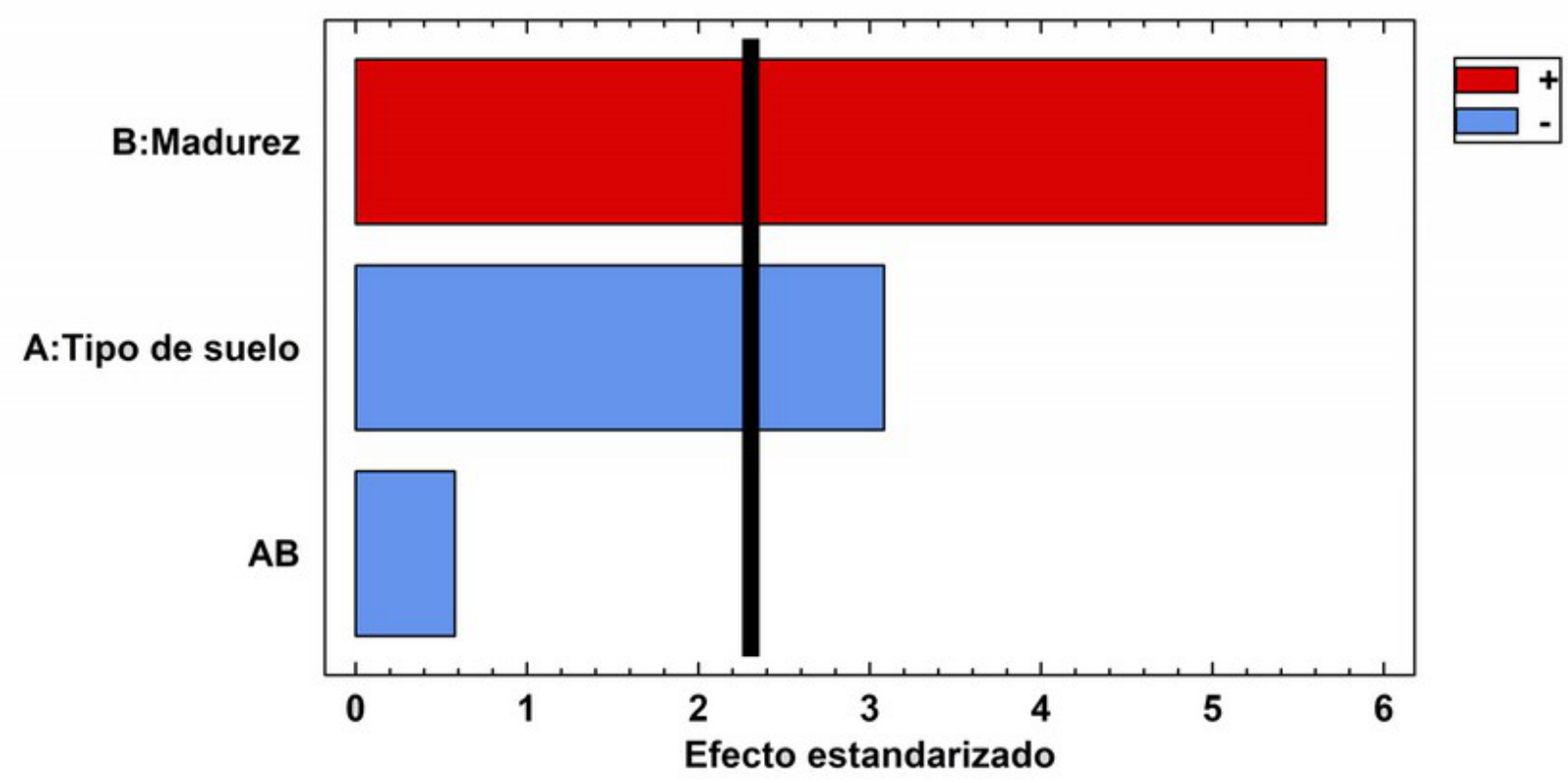

Figura 4. Diagrama de Pareto obtenido del diseño $3 \times 2$ para evaluar el efecto de los factores sobre la concentración de polifenoles totales.

El hecho que los factores tipo de suelo y grado de madurez tuvieran efecto significativo sobre la concentración de metabolitos secundarios se debe a que la biosíntesis de éstos suele estar restringida a estados específicos del desarrollo (como grado de madurez) y a periodos de estrés (generados por el tipo de suelo). El suelo rojo (K'ankab) se caracteriza por su bajo contenido de materia orgánica en comparación con otros suelos de la zona, y con la capacidad de retener menor humedad, estas características generan un mayor estrés en la planta, lo que pudiera originar una mayor producción de los metabolitos estudiados en el presente trabajo. ${ }^{7}$ 


\section{CONCLUSIONES}

El grado de madurez, fue el factor que tuvo efecto sobre todos los metabolitos analizados, mientras que, de tipo de suelo, tuvo efecto en todos excepto en la actividad antioxidante. Existió interacción de los dos factores (grado de madurez y tipo de tierra) para el contenido de capsaicina, dihidrocapsaicina, capsaicinoides totales y Vitamina C. El chile maduro (color naranja) cosechado de plantas crecidas en suelo rojo (K'ankab) fue el que tuvo los más altos contenidos de ácido ascórbico $(113.8 \pm 0.10 \mathrm{mg} / 100 \mathrm{~g})$, capsaicina $(319.36 \pm 0.00 \mathrm{mg} / 100 \mathrm{~g})$, dihidrocapsaicina $(389 \pm 0.00 \mathrm{mg} / 100 \mathrm{~g})$, polifenoles totales $(90.25 \pm 0.06 \mathrm{mg}$ $\mathrm{GAE} / 100 \mathrm{~g})$ y actividad antioxidante $(95.43 \pm 0.10 \%)$.

\section{AGRADECIMIENTOS:}

Al Consejo Nacional de Ciencia y Tecnología de México (CONACYT) por el financiamiento para la realización de este estudio como parte del proyecto No. 257588 y el autor Oney-Montalvo le agradece por la beca No. 703762 para la realización de sus estudios de doctorado.

\section{REFERENCIAS}

1. Medina, F.L.; Echeverria, I.M.; Pacheco, R.A.; Ruiz, N.L.; Guzman, A.; Martinez, M.E. (2008). Influence of Nitrogen and Potassium Fertilization on Fruiting Capsaicin Content in Habanero Pepper (Capsicum chinense Jacq.). Soil Management, Fertilization, and Irrigation, 43, 1549 - 1554. http://hortsci.ashspublications.org/content/43/5/1549.full

2. Cisneros P.; Torres, T.; Gutierrez P.; Contreras M.; Gonzalez E.; Peraza S. (2007). Capsaicinoids quantification in chili peppers cultivated in the state of Yucatan, Mexico. Food Chemistry, 104, 1755-1760. https://www.sciencedirect.com/science/article/pii/S0308814606008612

3. Borges, L.G.; Cervantes C.; Ruiz N.; Soria F.; Reyes O.; Villanueva C. (2010). Capsaicinoids in habanero pepper (Capsicum chinense Jacq.) under various 
humidity and nutritional conditions. Terra Latinoam, 28, $35-41$. http://www.scielo.org.mx/scielo.php?script=sci_arttext\&pid=S018757792010000100004

4. Tundis, R.; Loizzo, M.; Menichini, F.; Bonesi, M.; Conforti, F.; Statti, G.; De Luca, D.; de Cindio, B.; Menichini, F. (2011). Comparative study on the chemical composition, antioxidant properties and hypoglycaemic activities of two Capsicum annuum L. cultivars (Acuminatum small and Cerasiferum). Plant Foods Hum. Nutr, 66, 261-269. https://link.springer.com/article/10.1007/s11130-011-0248-y

5. Alvarez-Parrilla E, de la Rosa L, Amarowicz R, Shahidi F. (2011). Antioxidant activity of fresh and processed Jalapeno and Serrano peppers. J Agric Food Chem, 59 ,

$163-173$.

https://www.ncbi.nlm.n

6. Borges, G.L.; Moo, C.K.; Ruiz, J.N.; Osalde, M.B.; González, C.V.; Yam, C.C.; Can, F.P. (2014). Suelos destinados a la producción de chile habanero en Yucatán: características físicas y químicas predominantes. Agrociencia, 48, 347 - 359. http://www.scielo.org.mx/scielo.php?script=sci_arttext\&pid=S140531952014000400001

7. Bautista F, Jiménez J, Navarro J, Manu A, Lozano R (2003). Microrrelieve y color de suelos como propiedades distintivas. Terra, 23, $1-11$. https://www.redalyc.org/articulo.oa?id=57321101

8. Brand W, Cuvelier ME, Berset C (1995). Use of free radical method to evaluate antioxidant activity. LWT- Food Science and Technology, 28 (1) 25 - 30. https://www.sciencedirect.com/science/article/pii/S0023643895800085

9. Singleton, V.L.; Orthofer, R.; Lamuela-Raventos, R.M. (1999) Analysis of total phenols and other oxidation substrates and antioxidants by means of FolinCiocalteu reagent. Method Enzymol, 299, 152-178. https://www.sciencedirect.com/science/article/pii/S0076687999990171

10. Menichini, F.; Tundis, R.; Bonesi, M.; Loizzo, M.R.; Conforti, F.; Statti, G.; De Cindio, B.; Houghton, P.J.; Menichini, F. (2009). The influence of fruit ripening on the phytochemical content and biological activity of Capsicum chinense Jacq. cv Habanero, Food Chemistry, 114, 553-560. https://www.sciencedirect.com/science/article/pii/S0308814608011771 
11. Howard, L. R.; Talcott, S.T.; Brenes, C.H., A.B.; Villalon, B. (2000). Changes in phytochemical and antioxidant activity of selected pepper cultivars (capsicum species) as influenced by maturity. Journal of agriculture and food chemistry, 48, 1713 - 1720. https://pubs.acs.org/doi/abs/10.1021/jf990916t

12. Siddiqui, M.W.; Momin, C.M.; Acharya, P.; Kabir, J.; Debnath, M.K.; Dhua, R. S. (2013). Dhua Dynamics of changes in bioactive molecules and antioxidant potential of Capsicum chinense Jacq. cv. Habanero at nine maturity stages. Acta Physiol Plant, 35, 1141-1148. https://link.springer.com/article/10.1007/s11738012-1152-2

13. Mendoza, S.I.; Mendoza, M.R.; Barradas, O.G.; Nieto, E.A.; Pascual, L.A.; Fernández, M.J. (2015). Physicochemical and antioxidant properties of jalapeño pepper (Capsicum annuum var. annuum) during storage. Revista Chapingo Serie Horticultura, 21, 229 - 241. https://www.redalyc.org/html/609/60943322004/

Oney-Montalo J. ${ }^{1}$, López-Domínguez C. ${ }^{1}$, Zamacona-Ruiz M. ${ }^{1}$, Gómez-Rincón E. ${ }^{1}$, Ramíre-Sucrez M. ${ }^{1}$, Rodríguez-Buenfil I. ${ }^{{ }^{*}}$

${ }^{1}$ Centro de Investigación y Asistencia en Tecnología y Diseño del Estado de Jalisco (CIATEJ), Unidad Sureste. Parque Científico Tecnológico de Yucatán. Km5.5 Carr. Sierra Papacal-Chuburná Puerto. C.P. 97302.

*Autor de correspondencia: irodriguez@ciatej.mx 